\title{
Asteroid colors: a novel tool for magnetic field detection? The case of Vesta
}

\author{
P. Vernazza ${ }^{1}$, R. Brunetto ${ }^{2,3}$, G. Strazzulla ${ }^{3}$, M. Fulchignoni ${ }^{1}$, P. Rochette ${ }^{4}$, N. Meyer-Vernet ${ }^{1}$, and I. Zouganelis ${ }^{1,5}$ \\ 1 LESIA, Observatoire de Paris, 92195 Meudon Principal Cedex, France \\ e-mail: pierre.vernazza@obspm.fr \\ 2 Dipartimento di Fisica, Universita di Lecce, via Arnesano, 73100 Lecce, Italy \\ 3 INAF - Osservatorio Astrofisico di Catania, via Santa Sofia 78, 95123 Catania, Italy \\ 4 CEREGE CNRS Université d'Aix-Marseille 3, 13545 Aix en Provence Cedex 4, France \\ 5 Space Sciences Laboratory, University of California at Berkeley, Berkeley, CA 94720-7450, USA
}

Received 9 March 2006 / Accepted 30 March 2006

\section{ABSTRACT}

\begin{abstract}
Aims. Vesta's surface is surprisingly pristine. Although its basaltic surface is roughly similar to the lunar surface, which is intensely space weathered, its surface remains unaltered. It has been shown recently that solar-wind irradiation dominates asteroidal space weathering with a timescale on the order $10^{4}-10^{6}$ years. Recent ion irradiation experiments on pyroxenes have shown significant reddening and darkening of the collected spectra with progressive irradiation. Since pyroxene is a major surface component of Vesta as determined by spectroscopy, we aimed to test whether the solar wind irradiation alters significantly the optical properties of the surface of Vesta.

Methods. Consequently, we performed an ion irradiation experiment on a eucrite meteorite, which characterizes the surface of Vesta well, in order to simulate the solar wind irradiation on this asteroid.

Results. Our result implies that, if solar wind ions do reach the surface of Vesta, its reflectance spectrum should be much redder and its albedo lower. Indeed, this implies that solar wind particles can not have reached the asteroid surface. This strongly suggests the presence of a magnetic field shielding the surface from solar wind ions. This is the first remote detection of the magnetic field of an asteroid based on its color.
\end{abstract}

Key words. minor planets, asteroids - Moon - magnetic fields - laboratory - techniques: spectroscopic

\section{Introduction}

Space weathering processes affect Solar System bodies that are not protected by an atmosphere or a magnetosphere, altering the optical properties of their soil. The continuous bombardment by energetic cosmic rays, solar wind ions, and interplanetary dust particles (micro-meteorites) are the sources of this weathering. Such alteration processes change the spectral properties of silicate-rich objects, inducing progressive darkening and reddening of the solar reflectance spectra in the UV-Vis-NIR range (Hapke 2001; Sasaki et al. 2001; Brunetto \& Strazzulla 2005; Brunetto et al. 2006).

They have been studied in order to find a link between the spectral properties of meteorites and the remote sensing data of asteroids. Indeed, as demonstrated by laboratory experiments, space weathering can explain the spectral mismatch between the most populous class of meteorites (ordinary chondrites, OC) and the surface spectra of their presumed (S-type) asteroidal parent bodies (Pieters et al. 2000; Adams \& McCord 1971; Strazzulla et al. 2005; Marchi et al. 2005), while it also explain the spectral difference between lunar soils and underlying rocks. However, this spectral mismatch is not observed in the case of 4 Vesta (Chapman 2004), one of the three largest main belt bodies $(D=$ $529 \pm 10 \mathrm{~km}$ ) (Thomas et al. 1997). Vesta is the only known differentiated asteroid with an intact internal structure, probably consisting of a metal core, a mantle, and a basaltic crust (McCord et al. 1970).
Strong evidence suggests that the HED meteorites, a large set of differentiated basalts (Eucrites), pyroxenites (Diogenites), and breccia mixtures of mainly these two rock types (Howardites), are impact ejecta from Vesta. The link between HED meteorites and Vesta was originally based on the good matches between the reflectance spectra and brightness of Vesta (albedo $\sim 0.35$ ) and those of HED meteorites, indicating that Vesta's surface is free of heavy space weathering (Chapman 2004; McCord et al. 1970; Keil 2002; Gaffey 1983). On Vesta, the maturation effect due to micro-meteorite bombardment should be 100-1000 times lower than that of the Moon (Gaffey 1983); however, the alteration effects due to the solar wind on its surface have not been investigated so far. Recent ion irradiation experiments on pyroxenes have shown significant reddening and darkening of the collected spectra with progressive irradiation (Marchi et al. 2005). Since pyroxene is a major surface component of Vesta, as determined by spectroscopy, one could expect the solar wind irradiation to significantly alter the optical properties of the surface of Vesta.

\section{Experimental results}

To investigate this question, we irradiated with energetic ions a eucrite meteorite, Bereba, chosen because its reflectance spectrum is very similar to that of Vesta. The ion irradiation experiments were performed at the Observatory of Catania, using $\mathrm{Ar}^{++}$ions with energy of $400 \mathrm{keV}$ - higher than that of solar 


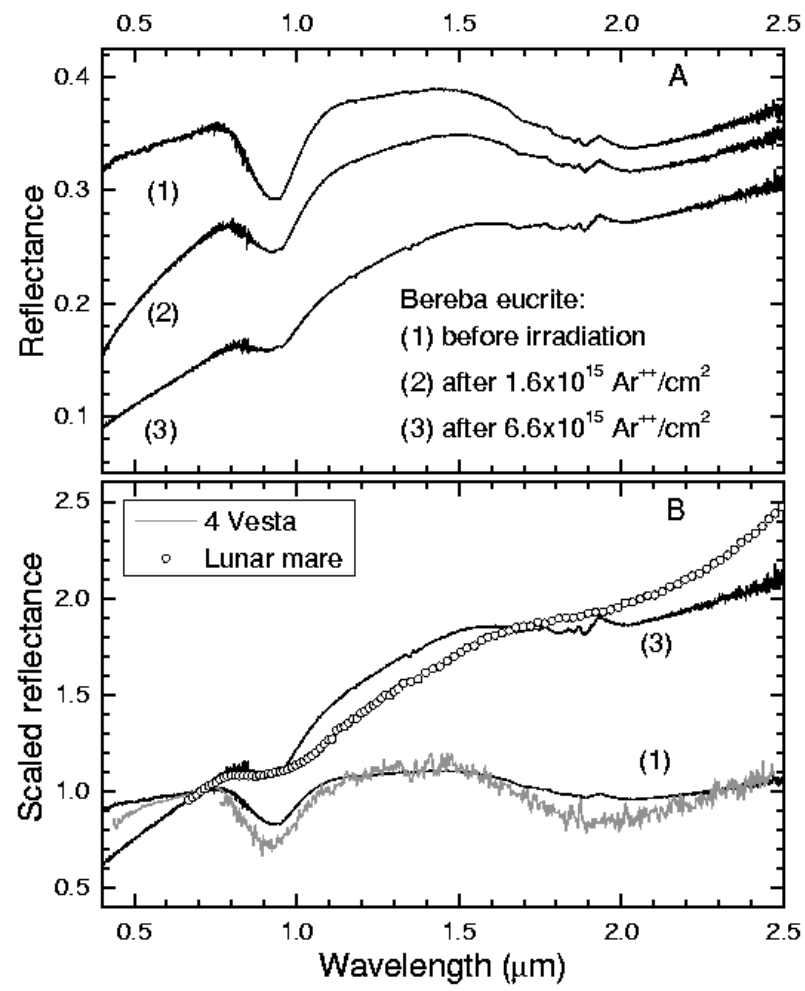

Fig. 1. a) VIS-NIR $(0.4-2.5 \mu \mathrm{m})$ reflectance spectra of the eucrite meteorite Bereba before and after irradiation with two different $\mathrm{Ar}^{++}$ion fluences. b) The initial reflectance spectrum of Bereba (1) and that obtained at the highest ion fluence (3) are shown scaled to 1 at $0.7 \mu \mathrm{m}$, and compared with the spectrum of Vesta and the spectrum of a small lunar mare area.

wind Ar ions ( $36 \mathrm{keV})$ - in order to accelerate the process, since it has been demonstrated that spectral alteration of silicates is determined by the energy deposited by incoming ions through elastic collisions with target nuclei (Brunetto \& Strazzulla 2005). We used $\mathrm{Ar}^{++}$ions rather than $1 \mathrm{keV}$ protons - by far the most abundant in the solar wind - since irradiation effects are much greater with heavy ions than with protons (Brunetto \& Strazzulla 2005). Other heavy ions have similar effects to argon (Strazzulla et al. 2005). Powdered $(10-100 \mu \mathrm{m})$ samples of Bereba were placed in a stainless steel vacuum chamber $\left(P<10^{-7}\right.$ mbar $)$ interfaced to a spectrophotometer (Bruker Equinox 55) through $\mathrm{KBr}$ windows and to an ion implanter (Danfysik 1080-200), from which ions with energy 10-200 keV (400 keV for double ionization) can be obtained. Ions produce a circular $(1.5 \mathrm{~cm}$ in diameter $)$ uniform spot on the target, and currents were set below $1 \mu \mathrm{A} \mathrm{cm}$ to avoid macroscopic heating of the sample. Reflectance spectra (Fig. 1a) were acquired using $\mathrm{BaSO}_{4}$ as the reflectance standard (Brunetto \& Strazzulla 2005).

The two bands in the spectra of Bereba around 0.9 and $2 \mu \mathrm{m}$ are due to pyroxene, while the small shoulder around $1.3 \mu \mathrm{m}$ is due to the presence of plagioclase. Irradiation of the sample produces a progressive darkening and reddening (i.e. lower reflectance at lower wavelength) of the spectrum. The spectral slope in the region of the first band (centered around $0.9 \mu \mathrm{m}$ ) increases strongly, while the depth of both absorption bands becomes weaker. Irradiation of a virgin sample of Bereba, whose spectrum and albedo are very close to those of Vesta, yields a spectrum that is very similar to the Moon's, in terms of spectral slope and albedo (Moon's albedo $\sim 0.1$ ) with a fluence of $6.6 \times$ $10^{15} \mathrm{Ar}^{++} / \mathrm{cm}^{2}$ (Fig. 1b). We converted fluences at five stages of

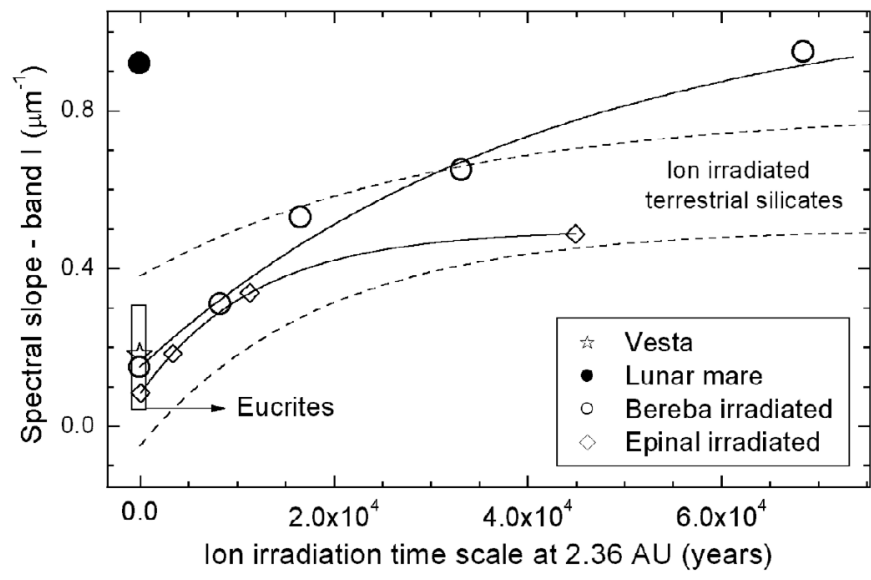

Fig. 2. Evolution of the spectral slopes with progressive irradiation as a function of time. The $x$-axis is the astrophysical timescale (at 2.36 AU) necessary to induce the observed spectral reddening on an asteroid surface. We include data from irradiation of Bereba, of the Epinal meteorite (with exponential fits to guide the eye), and of a number of terrestrial silicates (Brunetto \& Strazzulla 2005) rich in olivine and pyroxene. The spectral slope of Vesta and of the lunar mare area are also reported, as well as the range of spectral slopes for eucrites.

irradiation of Bereba to the corresponding timescale (Fig. 2) for the solar wind ions at 2.36 AU (average heliocentric distance of Vesta). The timescale is estimated by dividing the fluences used in the laboratory by the solar wind ion flux. This flux is the flux of $\mathrm{Ar}$ ions $\left(36 \mathrm{keV}\right.$ ) at $1 \mathrm{AU}$ (about $4 \times 10^{2}$ ions $\mathrm{cm}^{-2} \mathrm{~s}^{-1}$ ) scaled to $2.36 \mathrm{AU}$ with a variation as the inverse squared heliocentric distance. Then the timescale is corrected as follows. First, the time scale has to be multiplied by a factor of 6 , since the elastic collision damage of $400 \mathrm{keV}$ Ar ions is about 6 times stronger than $36 \mathrm{keV}$ Ar ions; second, we multiply by a factor of 4 to take into account the ratio between the geometrical cross-section and the whole surface assuming a spherical body uniformly irradiated. Third, since Ar is a minor component of the solar wind, we have to scale to all the other solar wind ions $(\mathrm{H}, \mathrm{He}, \mathrm{C}, \mathrm{O}, \mathrm{N}$, etc.), again using the elastic collision damage (the correction is about a factor of 1000).

The lunar Maria basalts and the Vesta crust are very similar in mineralogical composition (mainly pyroxene, plagioclase, and olivine). Even so, it appears that space weathering affecting the Moon surface minerals left Vesta's surface unalterated. In order to examine the effects of ion irradiation on silicates in more detail, we compared the spectral slope variations of Bereba with those obtained by irradiating the Epinal meteorite (H5 ordinary chondrite) (Strazzulla et al. 2005) and a number of terrestrial silicates (Brunetto \& Strazzulla 2005), rich in olivine and pyroxene (Fig. 2). The spectral slope is calculated from the spectra scaled to 1 at 0.7 microns, as the slope of a linear continuum across the 1 -micron band (from about $\lambda_{a} \sim 0.8 \mu \mathrm{m}$ to $\lambda_{b} \sim 1.5 \mu \mathrm{m}$ ), and is plotted versus the equivalent time in the solar wind at $2.36 \mathrm{AU}$.

The strong spectral effects observed with progressive irradiation are quite fast (timescales lower than $10^{5}$ years), and similar to previous experiments performed on silicates; Bereba reddens even faster than ordinary chondrite Epinal. Heavy particle irradiation of ordinary chondrite Epinal (Strazzulla et al. 2005) can explain the mismatch between ordinary chondrites and S-type asteroids and, most likely, solve this paradox. However, in the case of Vesta, the opposite paradox appears: if solar wind ions do reach the surface of Vesta, one should observe the same meteorite/asteroid spectral effect as for ordinary chondrites/S-type 
asteroids, but we did not. This implies that either Vesta is shielded from solar wind ions or some event refreshed the whole surface recently.

In the latter case, a large impact should have resurfaced the upper irradiated layers of the body less than about $10^{5}$ years ago (Fig. 2). Observations (Thomas et al. 1997) from the Hubble Space Telescope indicate that Vesta has a $460 \mathrm{~km}$ basin on its surface, which was the result of an impact from a $\sim 35 \mathrm{~km}$ projectile. Furthermore, it is likely that Vesta experienced only one such impact over the Solar System history (Bottke et al. 2005). Collisional modeling results suggest that this impact took place about $1 \mathrm{Gyr}$ ago and is responsible for the socalled Vestoids, a family of $D<10 \mathrm{~km}$ asteroids on Vesta-like orbits whose spectral features strongly resemble eucrites and howardites (Burbine et al. 2001; Marzari et al. 1996; Carruba et al. 2005). Considering that the Vestoids are not the result of this impact, Bottke et al. (2005) find a $19 \%$ probability that a major impact occurred on Vesta in the last 3.5 Gyr. Thus, a recent large impact (last $10^{5}$ years) on Vesta seems unlikely.

The remaining possibility is that Vesta's interior, or crust, is a good conductor or is sufficiently magnetized, with a sufficiently coherent magnetization direction, so that it will form a barrier to the solar wind (Greenstadt 1971). A remanent magnetic field is the most likely way of forming an obstacle to the solar wind flow resulting in its diversion. The present data do not enable us to distinguish between a global magnetic field producing a bona fide magnetosphere and a number of uniformly magnetized blocks of crustal material uniformly magnetized producing several crustal "magnetospheres". In the latter case, the solar wind particles would reach the surface via a number of "cusps".

To investigate this possibility, we estimated the fraction of Vesta's observed surface that must be unaltered, in order that Vesta's spectrum and albedo remain similar to those of HED meteorites. To make an order of magnitude estimate, we assumed that, if the solar wind ions touch the surface, the spectra of those unprotected regions would be similar to the most irradiated spectra of Bereba. By a linear combination of the most irradiated spectra of Bereba and the virgin spectra (geographical mixture), we found an upper limit of about $10 \%$ unprotected surface, over which it would be obvious that space weathering is occurring on Vesta; this limit is found when the slope of the linear combination is greater than the slope domain of eucrites. This estimate leaves both possibilities open.

For Vesta to block the solar wind from bombarding its surface, the pressure of its magnetic field must balance the solar wind ram pressure at a distance from the surface that is greater than the proton inertial length and their gyroradii in this magnetic field. Balancing the solar wind ram pressure of about $5 \times$ $10^{-10} \mathrm{~Pa}$ at $2.36 \mathrm{AU}$ requires that the asteroid produces a magnetic field $B_{0} \sim 2 \times 10^{-8} \mathrm{~T}$ at the stand-off distance. Since the above proton length scales $(\sim 200 \mathrm{~km})$ are within the same order of magnitude as the radius of Vesta, the radius of the "magnetosphere" at the subsolar point should be at least twice Vesta's radius. With a dipolar global magnetic field, decreasing with distance as $1 / r^{3}$, this requires a magnetic field strength of at least $2^{3} B_{0} \sim 0.2 \mu \mathrm{T}$ at the surface of Vesta - corresponding to a magnetic dipole $\sim 3 \times 10^{16} \mathrm{Am}^{2}$. Even though such a magnetic field is expected to stand-off the solar wind (Greenstadt 1971), this value is a lower limit since the size of the resulting magnetosphere is similar to the proton gyroradius, in which case kinetic effects are important (Omidi et al. 2002).

Alternatively, several large blocks of uniform magnetization within the crust, producing multiple crustal magnetospheres, would require higher surface magnetic fields to stand-off the solar wind and produce an irregularly shaped "magnetosphere". It is interesting to note that the Mars Global Surveyor spacecraft detected magnetic fields that imply crustal magnetized patches on Mars of magnetic moment within the same order of magnitude as the value estimated above, providing pressure responsible for localized increases in the ionopause altitude (Acuna et al. 1998). Such a mechanism has been demonstrated locally on the Moon by observed systematic coincidence between high albedo "swirls" and local magnetic field highs (Richmond et al. 2005). On the Moon, field strengths of only $10 \mathrm{nT}$ at $35 \mathrm{~km}$ altitude are sufficient for producing this effect.

In fact, geochemical and paleomagnetic evidence from the HED meteorites strongly suggests that Vesta formed an iron core and once had an internally generated magnetic field (Cisowski 1991; Collinson \& Morden 1994; Gattacceca \& Rochette 2004; Ruzicka et al. 1997; Righter \& Drake 1997). Eucrite and diogenite appear to have cooled in a field on the order of $10 \mu \mathrm{T}$, with on the average low remanence intensities on the order of $10 \mathrm{~mA} / \mathrm{m}$, due to low metal content. However, deeper rocks may have enhanced magnetization, as may regolith rocks with a higher metal content, which is demonstrated in howardite (Rochette et al. 2004). To generate a global field from a crust or mantle magnetized within a dynamo, one needs a non uniform crustal thickness (Aharonson et al. 2004). This type of coverage is exactly the situation of Vesta, lacking its crust over a large portion, corresponding to the large crater excavated at the present South pole (Thomas et al. 1997; Drake 2001). If the present rotation pole was the magnetic pole during remanence acquisition, this would generate a significant global remanent dipolar field.

In the case of the Moon - which has no global magnetic field or atmosphere - the average surface is exposed directly to the solar wind (except in the "swirls"), which plays an important role in the reddening and darkening of the lunar spectrum, in addition to micro-meteorite bombardment. Again, this suggests that Vesta may support a magnetic field that protects its surface from the solar wind.

\section{Conclusions}

1. Laboratory irradiation of the eucrite meteorite Bereba shows a comparable (even a bit stronger) space-weathering effect to terrestrial silicates and the ordinary chondrite Epinal.

2. Vesta's surface appears unaltered despite a short spaceweathering timescale derived from our experiment $\left(10^{5} \mathrm{yr}\right)$. Our result strongly suggests the presence of a magnetic field shielding a large portion of its surface from solar wind ions, with a required strength at the surface of $\sim 0.2 \mu \mathrm{T}$.

3. Just as Jupiter's magnetic field has been detected by remote sensing via its radio emission, long before space exploration, the present work provides a remote detection of Vesta's magnetic field via its color, opening the way to a novel technique of asteroid exploration.

Acknowledgements. We thank the Muséum National d'Histoire Naturelle for providing us with the meteoritic samples. The authors are grateful to G. A. Baratta, D. Fulvio, and F. Spinella for their precious support during the experiments. We thank the referee J. Emery for his pertinent and constructive remarks.

\section{References}

Acuna, M. H., Connerney, J. E. P., Wasilewski, P., et al. 1998, Science, 279, 1676 Adams, J. B., \& McCord, T. B. 1971, Science, 171, 567

Aharonson, O., Zuber, M. T., \& Solomon, S. C. 2004, Earth and Planetary Science Letters, 218,261 
Bottke, W. F. Durda, D. D., Nesvorný, D., et al. 2005, Icarus, 175, 111

Brunetto, R., Romano, F., Blanco, A., et al. 2006, Icarus, 180, 546

Brunetto, R., \& Strazzulla, G. 2005, Icarus, 179, 265

Burbine, T. H., Buchanan, P. C., Binzel, R. P., et al. 2001, Meteoritics and Planetary Science, 36,761

Carruba, V., Michtchenko, T. A., Roig, F., Ferraz-Mello, S., \& Nesvorný, D. 2005, A\&A, 441,819

Chapman, C. R. 2004, Annual Review of Earth and Planetary Sciences, 32, 539

Cisowski, S. M. 1991, Earth and Planetary Science Letters, 107, 173

Collinson, D. W., \& Morden, S. J. 1994, Earth and Planetary Science Letters, 126, 421

Drake, M. J. 2001, Meteoritics and Planetary Science, 36, 501

Gaffey, M. J. 1983, in Lunar and Planetary Institute Conference Abstracts, 231

Gattacceca, J., \& Rochette, P. 2004, Earth and Planetary Science Letters, 227, 377

Greenstadt, E. W. 1971, Icarus, 14, 374

Hapke, B. 2001, J. Geophys. Res., 106, 10039

Keil, K. 2002, Asteroids III, 573

Marchi, S., Brunetto, R., Magrin, S., Lazzarin, M., \& Gandolfi, D. 2005, A\&A, 443, 769
Marzari, F., Cellino, A., Davis, D. R., et al. 1996, A\&A, 316, 248

McCord, T. B., Adams, J. B., \& Johnson, T. V. 1970, Science, 168, 1445

Omidi, N., Blanco-Cano, X., Russell, C. T., Karimabadi, H., \& Acuna, M. 2002, J. Geophys. Res. (Space Phys.), 107, 12

Pieters, C. M., Taylor, L. A., Noble, S. K., et al. 2000, Meteoritics and Planetary Science, 35,1101

Richmond, N. C., Hood, L. L., Mitchell, D. L., et al. 2005, J. Geophys. Res. (Planets), 110, 5011

Righter, K. \& Drake, M. J. 1997, Meteoritics and Planetary Science, 32, 929

Rochette, P., Gattacceca, J., Menvielle, M., Eisenlohr, P., \& Chevrier, V. 2004, Planet. Space Sci., 52, 987

Ruzicka, A., Snyder, G. A., \& Taylor, L. A. 1997, Meteoritics and Planetary Science, 32, 825

Sasaki, S., Nakamura, K., Hamabe, Y, Kurahashi, E., \& Hiroi, T. 2001, Nature, 410, 555 Strazzulla, G., Dotto, E., Binzel, R., et al. 2005, Icarus, 174, 31

Thomas, P. C., Binzel, R. P., Gaffey, M. J., et al. 1997, Science, 277, 1492 